\section{PERCEPÇÃO DOS PROFISSIONAIS SOBRE A ESTRATÉGIA SAÚDE DA FAMÍLIA EM UM MUNICÍPIO DO INTERIOR DO CEARÁ}

\author{
Professionals' perception of the Family Health Strategy in an \\ inland city in the state of Ceará
}

\author{
Percepción de los profesionales sobre la Estrategia de Salud \\ de la Familia de un municipio del interior de Ceará
}

\begin{abstract}
RESUMO
Objetivo: Conhecer a percepção dos profissionais sobre a Estratégia Saúde da Família (ESF) em um município do interior do Ceará. Métodos: Estudo descritivo, de natureza qualitativa, realizado de fevereiro a abril de 2015 com profissionais de saúde de sete Unidades Básicas de Saúde de Limoeiro do Norte, Ceará e um gestor coordenador da atenção básica do município. As entrevistas foram gravadas em local reservado e data agendada previamente. A análise das informações ocorreu através da análise de conteúdo. Identificaram-se as seguintes categorias: "Implementação da ESF", "Caracterização das ações da ESF", "Percepção sobre a ESF pelos atores" e "Dificuldades encontradas para implementação da ESF". A pesquisa teve aprovação do Comitê de Ética em Pesquisa da Universidade da Integração Internacional da Lusofonia Afro-Brasileira (UNILAB), de acordo com a Resolução 466/12. Resultados: O gestor não tinha clareza do processo de implementação da Estratégia no município. Destacou a relação de confiança entre profissionais e população para que a equipe seja bem aceita na comunidade e a importância de um fluxo de atenção no serviço público de saúde. Os profissionais relataram a satisfação do trabalho em equipe, a boa comunicação entre os membros e acreditam que têm formação necessária para trabalhar na atenção básica. Ressaltaram, ainda, o reconhecimento da população pelo trabalho executado, mas reclamaram da falta de apoio da gestão do município em fornecer insumos para o trabalho. Conclusão: Os participantes acreditam na importância do trabalho em equipe para minimizar os problemas da estratégia, mas apresentaram-se desestimulados com a atual situação da saúde do município.
\end{abstract}

Descritores: Estratégia Saúde da Família; Pesquisa Qualitativa; Entrevista; Profissional da Saúde.

\section{ABSTRACT}

Objective: To assess the profissional's perception of the Family Health Strategy in an inland city of the state of Ceará. Methods: Descriptive study of qualitative approach, conducted from February to April 2015 with health professionals from seven Primary Health Units of Limoeiro do Norte, Ceará and a municipal primary care coordinator. The interviews were recorded in a private place on a previously scheduled date. The information was analyzed through content analysis. The following categories were identified: "Implementation of the Strategy"; "Characterization of the actions of the Strategy"; "Perception of the Strategy by the actors"; and "Difficulties encountered in the Strategy implementation". The research was approved by the Research Ethics Committee of the University for International Integration of the Afro-Brazilian Lusophony (UNILAB), according to Resolution 466/12. Results: The coordinator had not a clear knowledge of the Strategy implementation process in the city. He highlighted the necessary trusting relationship between professionals and the population for the team to be well accepted in the community, and the importance of a stream of attention in the public health service. Professionals reported satisfaction with the teamwork and good communication between the team members, and they believe they have the necessary training to work in primary care. Additionally, they highlighted the population's recognition of the work performed, but complained about the lack of support from the municipal management
Artigo Original

1) Universidade da Integração Internacional da Lusofonia Afro-Brasileira - UNILAB Redenção (CE) - Brasil

2) Secretaria de Saúde do Estado do Ceará SESA - Fortaleza (CE) - Brasil

3) Universidade Estadual do Rio Grande do Norte - UERN - Natal (RN) - Brasil

Recebido em: 07/07/2015 Revisado em: 28/10/2015 Aceito em: 28/11/2015 
on the provision of inputs for the activities. Conclusion: The participants believe in the importance of teamwork to minimize the Strategy problems, but presented themselves discouraged with the current health situation of the municipality.

Descriptors: Family Health Strategy; Qualitative Research; Interview; Health Personnel.

\section{RESUMEN}

Objetivo: Conocer la percepción de los profesionales sobre la Estrategia de Salud de la Familia (ESF) de un municipio del interior de Ceará. Métodos: Estudio descriptivo, de abordaje cualitativo realizado entre febrero y abril de 2015 con profesionales de la salud de siete Unidades Básicas de Salud de Limoeiro do Norte, Ceará y un gestor coordinador de la atención básica del municipio. Las entrevistas fueron grabadas en un sitio reservado y fecha definida con antelación. El análisis de las informaciones se dio a través del análisis de contenido. Se identificaron las siguientes categorias: "Implementación de la ESF", "Caracterización de las acciones de la ESF”, "Percepción de la ESF en la opinión de los actores" y "Dificultades encontradas para la implementación de la ESF". La investigación fue aprobada por el Comité de Ética en Investigación de la Universidad de la Integración Internacional de la Lusofonia Afro-Brasileña (UNILAB) según la Resolución 466/12. Resultados: El gestor no estaba seguro del proceso de implementación de la Estrategia del municipio. Se destaca la relación de confianza entre los profesionales y la población para que el equipo sea bien recibido por la comunidad y la importancia de un flujo de atención en el servicio público de salud. Los profesionales relataron la satisfacción del trabajo en equipo, la buena comunicación de los miembros y creen tener la formación necesaria para el trabajo en la atención básica. Resaltaron aún el reconocimiento de la población por el trabajo realizado pero reclamaron de la falta de apoyo de la gestión del municipio en ofrecer insumos para el trabajo. Conclusión: Los participantes creen en la importancia del trabajo en equipo para disminuir los problemas de la estrategia pero se presentaron sin estimulo delante la situación actual de la salud del municipio.

Descriptores: Estrategia de Salud Familiar; Investigación Cualitativa; Entrevista; Personal de la Salud.

\section{INTRODUÇÃO}

O Ministério da Saúde propôs a idealização da Estratégia Saúde da Família (ESF), com o propósito de reorganizar o modelo assistencial a partir da atenção básica. Sua finalidade é organizar os serviços e orientar práticas profissionais visando à promoção da saúde e prevenção de doenças ${ }^{(1)}$.

Na realidade econômica da distribuição de renda do país, hoje, a maioria dos usuários da ESF é formada por pessoas com nível aquisitivo baixo. As classes emergentes, por não acreditarem na eficácia da saúde pública, estão procurando cada vez mais alternativas em atendimentos de saúde, como convênios e planos de saúde ${ }^{(2)}$.

Os principais motivos da evasão dos usuários são o difícil acesso ao sistema, a demora no atendimento e na realização de procedimentos, e a falta de atendimento humanizado, sendo cruciais para o distanciamento da população do serviço e reclamações sobre este ${ }^{(3,4)}$.

Os profissionais da ESF são reconhecidos como os operadores do serviço, responsáveis pelo fluxograma desenvolvido e detentores do conhecimento técnico utilizado nas práticas ambulatoriais. Sendo assim, eles são fonte preciosa de informação sobre o sistema, pois, além de viverem o cotidiano da unidade, também são capazes de modificá-la para melhor atender os usuários, favorecendo a ampliação de ações de promoção da saúde e prevenção de doenças ${ }^{(5)}$.

Ressalta-se, porém, que uma das maiores dificuldades de implementação da ESF é a carência de profissionais com formação generalista, que atuem de forma efetiva na complexa demanda da atenção básica ${ }^{(6)}$. Cabe aos gestores do Sistema Único de Saúde (SUS) desenvolver estratégias que minimizem os problemas encontrados por esses profissionais $^{(7)}$.

Por outro lado, o excesso de rotatividade dos gestores municipais de saúde, fato comum em municípios do interior, põe em risco a continuidade do cuidado e das ações realizadas pelo gestor anterior. Os gestores da saúde de municípios menores se veem cada vez mais cobrados, devido à elevada demanda por consultas de especialidades, procedimentos e cirurgias, tanto pelos usuários quanto pelos profissionais de saúde, principalmente médicos ${ }^{(8)}$.

A preocupação em garantir a qualidade da saúde torna-se cada vez mais debatida entre os profissionais. A exigência pela qualidade, tanto por parte da gestão quanto dos usuários, é percebida no processo de trabalho diário e faz com que o aprimoramento das práticas seja cada vez mais importante ${ }^{(9)}$.

O presente estudo se justifica porque a qualidade da assistência ao usuário é o objetivo principal de todas as ações realizadas. Há poucos estudos na literatura sobre as percepções dos profissionais da saúde e gestores municipais acerca da ESF, bem como ainda não há uma avaliação desse programa utilizando esses dois atores do SUS no interior do estado do Ceará. Dessa forma, é de extrema importância conhecer a realidade política local, a fim de auxiliar os gestores a definir estratégias que visem solucionar possíveis problemas que ocorram nessa região.

O objetivo do estudo foi conhecer a percepção dos profissionais sobre a Estratégia Saúde da Família (ESF) em um município do interior do Ceará. 


\section{MÉTODOS}

Estudo descritivo e de natureza qualitativa, realizado no período de fevereiro a abril de 2015 com profissionais de saúde (médicos, enfermeiros e técnicos de enfermagem) que trabalham nas sete Unidades Básicas de Saúde (UBS), localizadas na zona urbana da cidade de Limoeiro do Norte, Ceará, além de um gestor responsável pela coordenação da atenção básica do município.

Os critérios de inclusão consistiram em: ser profissional da saúde, atuar nas UBS da zona urbana do município e concordar em participar do estudo. Excluíram-se os profissionais que tiveram incompatibilidade de agenda, encontravam-se de férias, estavam doentes no período da coleta de dados ou que se recusaram a participar do estudo. A fonte de coleta de dados foi primária, junto aos profissionais e gestor de saúde, seguindo os critérios de inclusão e exclusão estabelecidos.

Inicialmente, 26 profissionais participariam do estudo, porém, oito foram excluídos, totalizando 18 participantes.

Utilizaram-se dois roteiros de entrevista adaptados ${ }^{(10)}$ um para os profissionais da assistência e outro para o gestor. Para os profissionais da assistência, o roteiro continha dados de identificação (sexo, formação e tempo de atuação na UBS) e questões que contemplaram: experiências anteriores em outros locais de trabalho; se acreditavam que tinham formação adequada para o trabalho; razões que os levaram a trabalhar na ESF; as atribuições no cargo que ocupam; como avaliam o trabalho que desenvolvem na comunidade, sua equipe de trabalho e na ESF; que critérios definiriam uma boa equipe de saúde da família; como são tomadas as decisões; grau de satisfação com o trabalho que desenvolvem; pontos positivos e negativos do trabalho; as principais dificuldades do trabalho; e se sentem que seu trabalho é valorizado.

O roteiro para o gestor continha dados de identificação (sexo, formação e tempo de atuação no cargo) e questões que contemplaram: a que se propõe a ESF; as atribuições no cargo de gestor; que critérios definiriam uma boa equipe de saúde da família; critérios de escolha dos profissionais da ESF; se a ESF era bem aceita pela comunidade; benefícios e dificuldades da ESF no município estudado.

Os indivíduos investigados obtiveram informações quanto aos objetivos e metodologia do estudo, bem como tiveram a garantia do anonimato por ocasião dos achados da pesquisa. Os profissionais que aceitaram participar da pesquisa assinaram o Termo de Consentimento Livre e Esclarecido (TCLE), e o gestor assinou o TCLE e a carta de anuência, a fim de consentir com a realização da pesquisa em repartições públicas municipais. A identidade dos entrevistados foi mantida em sigilo.

As fontes básicas de dados consistiram nos depoimentos gerados a partir das entrevistas gravadas. Os participantes que não aceitaram o uso do gravador tiveram suas respostas anotadas na íntegra pelos investigadores. A entrevista aconteceu no ambiente de trabalho, em um local reservado, onde não existia trânsito de pessoas nem barulho, com data agendada previamente junto aos sujeitos do estudo.

Após a coleta de dados, procedeu-se à organização das falas dos sujeitos da pesquisa. As entrevistas passaram por uma leitura flutuante sobre os temas mais relevantes.

Para a organização dos depoimentos, utilizou-se a análise de conteúdo, mais especificamente a fase de categorização $^{(11)}$. Essa opção metodológica permite penetrar no mundo dos significados das ações e relações humanas, um lado não perceptível e captável em equações, médias, gráficos e medidas estatísticas ${ }^{(12)}$. Realizou-se a apreciação e agregação das respostas convergentes, utilizando o tema como unidade de registro. Em seguida, realizou-se a classificação e o agrupamento dos elementos que constituem cada unidade temática, extraída dos depoimentos dos participantes, a partir da interpretação dos dados colhidos, por analogia, e organizando-os por categorias, ou seja, através da parte comum dos dados existentes ${ }^{(11)}$. Os resultados foram confrontados com a literatura existente no âmbito nacional sobre os assuntos vigentes no inquérito científico.

Identificaram-se as seguintes categorias: "Implementação da ESF", "Caracterização das ações da ESF", "Percepção sobre a ESF pelos atores" e "Dificuldades encontradas para implementação da ESF".

A pesquisa foi aprovada pelo Comitê de Ética em Pesquisa da Universidade da Integração Internacional da Lusofonia Afro-Brasileira (UNILAB), sob o parecer $n^{\circ}$ 901.251, respeitando a Resolução 466/12 do CNS/ MS, referente a estudos envolvendo seres humanos. $\mathrm{Na}$ apresentação dos dados, os participantes foram denominados por profissional 1, profissional 2, e assim sucessivamente, como meio de preservação do anonimato.

\section{RESULTADOS E DISCUSSÃO}

A caracterização dos participantes do estudo encontrase na Tabela I. A maioria dos profissionais entrevistados era de mulheres formadas na área da enfermagem, com mais de 10 anos de formação profissional e de atuação na ESF, mas que atuavam na UBS em que estão atualmente há menos de 5 anos. A maior parte dos profissionais de nível superior tinha pós-graduação, e nenhum técnico de enfermagem havia iniciado um curso superior.

A partir dos resultados, foi possível expor algumas reflexões sobre a ESF no município pesquisado. Verificase o predomínio de mulheres dentro do serviço da ESF, o que demonstra a feminilização da força de trabalho na saúde pública do Brasil. Esse mesmo dado foi encontrado 
Tabela I - Caracterização dos profissionais de saúde participantes do estudo. Limoeiro do Norte, Ceará, 2015.

\begin{tabular}{|c|c|c|}
\hline Variáveis & $\mathrm{n}(\mathrm{n}=17)$ & $\%$ \\
\hline \multicolumn{3}{|l|}{ Sexo } \\
\hline Masculino & 01 & 5,88 \\
\hline Feminino & 16 & 94,12 \\
\hline \multicolumn{3}{|l|}{ Formação } \\
\hline Enfermeiro & 08 & 47,06 \\
\hline Técnico de enfermagem & 06 & 35,29 \\
\hline Médico & 03 & 17,65 \\
\hline \multicolumn{3}{|l|}{ Tempo de atuação profissional } \\
\hline$<5$ anos & 03 & 17,65 \\
\hline 5 a 10 anos & 01 & 5,88 \\
\hline$>10$ anos & 13 & 76,47 \\
\hline \multicolumn{3}{|l|}{ Tempo de atuação no PSF } \\
\hline$<5$ anos & 06 & 35,29 \\
\hline 5 a 10 anos & 03 & 17,65 \\
\hline$>10$ anos & 08 & 47,06 \\
\hline \multicolumn{3}{|l|}{ Tempo de atuação na UBS } \\
\hline$<5$ anos & 11 & 64,71 \\
\hline 5 a 10 anos & 02 & 11,76 \\
\hline$>10$ anos & 04 & 23,53 \\
\hline \multicolumn{3}{|c|}{ Pós-graduação (para enfermeiros e médicos) (n=11) } \\
\hline Sim & 10 & 90,91 \\
\hline Não & 01 & 9,09 \\
\hline \multicolumn{3}{|c|}{ Escolaridade (para técnicos de enfermagem) $(n=6)$} \\
\hline Ensino médio completo & 06 & 100 \\
\hline Ensino superior incompleto & - & - \\
\hline Ensino superior completo & - & - \\
\hline
\end{tabular}

n: número da amostra correspondente; \%: percentual da amostra correspondente; ESF: Estratégia Saúde da Família; UBS: Unidade Básica de Saúde.

em outras pesquisas relacionadas ao tema ${ }^{(13)}$. Em algumas profissões, esse processo de feminilização é mais recente e de forte impacto, como é o caso dos médicos: na década de 1970, as mulheres médicas eram apenas $11 \%$; já na década seguinte, esse percentual elevou-se para $22 \%$, chegando aos anos 1990 a 33\%. Estima-se que nas próximas décadas esse percentual deve atingir $50 \%{ }^{(14)}$.

Em um estudo que abordou o mesmo público-alvo, a maioria dos profissionais entrevistados também trabalhava há menos de 5 anos na $\mathrm{UBS}^{(10)}$. Quanto maior o tempo de atuação dos profissionais da atenção básica, maior é a convivência, formação de vínculos, comunicação, confiança $\mathrm{e}$, consequentemente, maior a resolubilidade dos casos do território $^{(15)}$.

Outro aspecto observado nessa mesma pesquisa foi que a maioria dos profissionais de nível superior também tinha pós-graduação ${ }^{(10)}$. Estudo verificou que os profissionais de saúde estão se especializando cada vez mais ao longo dos anos, e não só os profissionais que compõem a ESF, mas também outras novas profissões que têm se inserido na equipe de saúde ${ }^{(16)}$. É de extrema importância que os profissionais da atenção básica se especializem na área de atuação, por ser um serviço muito dinâmico e, por estar em constante renovação, necessitar de capacitações frequentes e estímulo à educação permanente ${ }^{(13)}$.

Quanto aos gestores públicos municipais, só foi possível realizar o estudo com uma mulher, que era a coordenadora da atenção básica do município. Ela tinha apenas formação em enfermagem (sem pós-graduação) e pouca experiência na área de gestão (apenas quatro meses). O tempo de atuação de um gestor de saúde é relevante para o desempenho do seu trabalho, pois a vivência com 
as atividades cotidianas do serviço vai propiciar uma maior execução no planejamento, organização, direção e controle do trabalho ${ }^{(14)}$.

Os profissionais da saúde que não se especializam na área de gestão para assumir um cargo desse porte acabam por não desenvolver as atividades administrativas adequadamente $^{(17)}$. A especialização na área de gestão favorece o desenvolvimento de atividades específicas, levando-se em consideração que a formação em planejamento ou em saúde pública imprime mais qualidade à atividade, uma vez que o planejamento constitui área básica de conhecimento para a formação de profissionais nessas áreas ${ }^{(18)}$.

As informações foram organizadas conforme as seguintes categorias: "Implementação da ESF", "Caracterização das ações da ESF", "Percepção sobre a ESF pelos atores" e "Dificuldades encontradas para implementação da ESF".

\section{Implementação da ESF}

Verificou-se que a profissional encarregada de gerir a saúde no município não tinha clareza de como se dava localmente o processo de implementação da ESF. Isso demonstra sua falta de conhecimento quanto aos acontecimentos históricos alusivos à saúde do município pelo qual é responsável. Esse dado pode estar atrelado ao pouco tempo de atuação no cargo de gestão (apenas 4 meses), bem como à pouca experiência profissional (formada há 2 anos).

No que diz respeito ao motivo pelo qual essa estratégia foi criada, a gestora identificou a ESF como sendo um laço que deixa o serviço público de saúde mais próximo da população, a fim de suprir demandas existentes até mesmo nos locais mais carentes de atenção. Quando perguntada sobre o propósito da ESF, respondeu:

\section{"Prestar assistência integral à população, buscando satisfazer as necessidades da mesma. Estruturar os serviços de saúde tanto para a relação com a comunidade como para os diversos niveis de assistência (...)." (GESTORA)}

Relato semelhante foi encontrado em um estudo em que gestores entrevistados citaram como características mais importantes do ESF a educação em saúde, a assistência e a promoção da saúde da população, o que revela uma perspectiva do que seja a proposta do programa ${ }^{(10)}$.

Com relação à implementação da ESF, é de suma importância a elaboração de políticas e ações de saúde que tenham maiores chances de se tornar efetivas e tragam maiores benefícios para a saúde da população ${ }^{(19)}$.
A gestora ressaltou a importância dada ao elo profissionais/comunidade para a permanência de laços fortes e raízes bem fincadas, para que a ESF fosse bem aceita e a assiduidade da população ao serviço fosse contínua e duradoura. Falou, inclusive, sobre o estabelecimento de laços de amizade, que favoreciam um maior vínculo entre as partes. Quando questionada sobre o que era mais importante na ESF, respondeu:

"O vínculo e a confiança (...) que deve (sic) existir entre a comunidade e os profissionais da unidade." (GESTORA)

Essa fala corrobora com o que foi mostrado em um estudo no qual os entrevistados ressaltaram a importância de os profissionais terem comprometimento com o serviço prestado, bem como serem acessíveis e atentos às necessidades da população ${ }^{(10)}$.

Em outra fala, a gestora deu muita importância à capacidade resolutiva da atenção primária em saúde, evitando, assim, que determinados casos fossem encaminhados indevidamente para outro serviço sem que haja uma real necessidade. Sobre os benefícios da ESF para a população, a gestora referiu:

"A porta de entrada para um paciente deve ser o PSF, tendo possivelmente a solução, e não indo para um hospital resolver (...) uma coisa simples." (GESTORA)

Gestores entrevistados em Minas Gerais também ressaltaram que o maior benefício que a ESF proporciona à população é a intervenção cada vez mais próxima e junto às famílias ${ }^{(10)}$.

A capacidade dos serviços de saúde de resolver os problemas é limitada tanto por causa de sua natureza, que exige intervenções de vários tipos, quanto por causa da sua ineficácia, evidenciada pelas avaliações científicas disponíveis ${ }^{(20)}$.

Pode-se notar que, para a gestora, o fluxo do paciente ao longo do serviço público de saúde prestado é algo a ser levado em consideração, uma vez que a ida do paciente a setores indevidos de assistência poderá acarretar mais custos para os cofres públicos. Ao ser questionada sobre a organização e encaminhamentos da ESF para outros níveis de assistência, disse:

"Através de uma ficha de referência, onde nela contém os dados pessoais do paciente, motivo do encaminhamento, resultado do exame e para qual especialidade está sendo encaminhado." (GESTORA)

A descentralização do SUS possibilitou que os municípios assumissem a responsabilidade de organizar fluxos, estruturas e processos de gestão para melhorar, 
organizar e expandir a atenção à saúde. A criação da ficha de referência pode ser vista como um desses instrumentos. Ela se materializou a partir da necessidade de regular o acesso da população entre os setores da atenção à saúde ${ }^{(21)}$.

Os gestores precisam de autonomia para adaptar os serviços às necessidades específicas dos usuários e fazer com que cada serviço seja da melhor qualidade para a população e tenha o melhor custo-benefício possível para a gestão(18).

\section{Profissionais de saúde}

As trajetórias dos profissionais de saúde foram apresentadas nesta categoria, por considerarmos que elas influenciam diretamente na implementação da ESF.

Quando indagados sobre uma formação adequada para o trabalho que desenvolve no PSF, a maioria dos profissionais da saúde respondeu que teve uma formação específica, voltada para o trabalho no programa, bem como experiências anteriores em outras áreas.

"Felizmente, a minha faculdade era muito voltada para a saúde pública, muito voltada para a atenção básica, para o PSF." (ENFERMEIRA 7)

"Sim. Trabalhei em Cuba e na Venezuela com clínica geral e medicina familiar." (MÉDICO 1)

Já ao avaliar as principais razões para trabalhar no PSF, a grande parte dos entrevistados respondeu que era por gostar de trabalhar diretamente com os pacientes, e também ressaltaram o amor de trabalhar com a comunidade.

"Eu gosto dessa relação, eu gosto do vínculo, de conhecer o paciente, de ter aquele vínculo, apesar de dar muito mais trabalho (...). Eu gosto muito dessa relação com a população. Eu acho melhor do que aquela prática de que você vai lá, dá plantão, vira as costas e não tem mais vínculo nenhum com aquele paciente. Eu não, eu gosto assim, do dia a dia. Me faz bem assim, de uma certa forma, então eu acho que o principal motivo foi esse." (ENFERMEIRA 4)

"Vocação. Gosto do trabalho e do atendimento da família." (MÉDICO 1)

Essas informações não corroboram com uma pesquisa realizada em Minas Gerais, na qual os autores observaram que o principal motivo pelo qual os profissionais de saúde estavam na ESF era a maior oferta de trabalho ${ }^{(10)}$. Podemos perceber a importância desse resultado, pois a relação das equipes com as famílias é o foco central da saúde da família, já que os profissionais de saúde da família devem, antes de tudo, ser comprometidos com seus usuários. Estudos demonstram que a atenção básica organizada pela ESF, quando bem capacitada e integrada à comunidade, é capaz de resolver $85 \%$ das demandas de saúde da população( ${ }^{(22)}$.
No que se refere ao que é necessário para desempenhar bem as suas funções, a maioria respondeu que o estudo e a especialização são essenciais.

"Ter uma boa formação e ter cursos que capacita a gente; a gente ter disponibilidade para desempenhar um bom trabalho e ter subsidios (...)." (TECNICA DE ENFERMAGEM 4)

"Uma boa formação, o gostar, o prazer, sentir-se bem atendendo à população. E, de uma forma geral, a gente ser bem gratificado também, porque isso concorre ao seu prazer, né?" (MÉDICO 2)

A ESF deve se apropriar e reformular o saber em saúde para que seus objetivos e diretrizes possam ser cumpridos de maneira satisfatória. Sendo assim, é de fundamental importância a sua formação, a instituição de programas de educação permanente, com cursos, discussões de casos e conselhos clínicos que tornem possível a conclusão aceitável desse trajeto $^{(10)}$.

Há problemas sérios com a política de pessoal, desde o sistema precário de contratação até a quase inexistência de oportunidade tanto para a formação especializada quanto para acesso a processos de educação permanente. A maioria absoluta dos médicos e enfermeiros não tem formação especializada em saúde da família, saúde coletiva ou para o exercício de uma clínica ampliada de cunho generalista, tampouco contam com apoio técnico ou institucional ${ }^{(23)}$.

A ESF é tida como uma área de trabalho nova em vários aspectos, principalmente no que se refere às experiências anteriores dos membros das atuais equipes, por isso, apresenta-se como um desafio técnico, por representar uma lógica operativa diferente da que os profissionais de saúde estavam, até então, habituados ${ }^{(24)}$, como citado por uma das entrevistadas:

"Na época de minha conclusão, não tinha PSF, portanto, fui aprendendo com o tempo e com a especialização." (ENFERMEIRA 1)

Ainda explorando esse mesmo assunto, apesar da importância do trabalho clínico, uma profissional de saúde, quando indagada sobre como é o seu processo de trabalho, destacou mais acentuadamente a importância da prática preventiva como sendo um dos pilares norteadores da atenção básica:

"Acredito que seja contínuo e de prevenção, já que trabalhamos na área de atenção primária." (ENFERMEIRA 3)

Essa mesma perspectiva foi encontrada no estudo mineiro ${ }^{(10)}$. No entanto, deve-se avaliar como essa prevenção vem sendo realizada por essas equipes, visto que ela representa apenas um dos pressupostos da ESF, ou seja, pelo 
fato de se prender a uma estratégia integral, deve associar assistência, prevenção e promoção à saúde ${ }^{(25)}$.

Outro ponto analisado foram os processos de trabalho em equipe e as relações interpessoais. Na avaliação do trabalho em equipe, a maioria avaliou as relações como sendo boas ou ótimas. Mas uma das entrevistadas usou o Programa Nacional de Melhoria ao Acesso e da Qualidade da Atenção Básica (PMAQ) para fazer sua avaliação:

\begin{abstract}
"No PMAQ, nós tiramos "excelente". A gente é suspeito em falar, mas o PMAQ nos deu essa resposta. Foi uma das equipes que tirou "excelente", e eles fazem entrevista ao usuário, e então é o usuário que tem que nos dar essa resposta. A equipe toda se coloca muito bem diante da população, muito embora às vezes tem algumas falhas, mas no geral eu acho que ela está muito bem posicionada, muito bem desenvolvida no que ela faz." (ENFERMEIRA 4)
\end{abstract}

O governo federal, procurando melhorar o padrão de qualidade do atendimento nas UBS, criou o PMAQ. Para isso, os profissionais que fazem parte de uma equipe de saúde (médicos, enfermeiros, dentistas, técnicos de enfermagem, agentes de saúde bucal e agentes comunitários de saúde) são acompanhados e avaliados. Essa avaliação leva em consideração a infraestrutura das UBS, os equipamentos, a disponibilização de medicamentos e a satisfação do cidadão. As equipes que oferecem melhorias na qualidade do atendimento recebem mais recursos do governo federal. Ou seja, quanto melhor for o desempenho, mais incentivos financeiros serão repassados ${ }^{(26)}$.

Já quando se perguntou sobre o que define uma boa equipe de saúde da família, as opiniões divergiram muito. Alguns destacaram a união e uma equipe atuante, outros destacaram a presença de uma equipe completa:

"Em primeiro lugar, a inter-relação entre todos nós. A comunicação é muito importante.” (MÉDICO 1)

"Pra ter uma equipe boa (...), é bom que tivesse todos os profissionais no PSF. (...) Mas, assim como te falei, falta mais disponibilidade, falta mais uma pessoa da equipe pra me ajudar. (...) Tá faltando (...) técnico em saúde bucal também, que a gente ainda não tem." (TÉCNICA DE ENFERMAGEM 4)

Nessa dimensão, um estudo mostrou que questões relacionadas ao número insuficiente de profissionais e a sobrecarga de trabalho levam a uma fragmentação dos processos de trabalho em equipe, de momentos coletivos, do perfil e da responsabilização profissional ${ }^{(27)}$. Outro estudo aponta que o número insuficiente de profissionais nas equipes de saúde tem dificultado o acesso aos serviços e o acolhimento aos usuários ${ }^{(28)}$. Desse modo, com uma demanda expressiva de atendimentos, ocorre uma sobrecarga de trabalho, dificultando as relações entre a equipe e os usuários ${ }^{(29)}$.

No tocante à avaliação do trabalho do médico na equipe, a maioria destacou que ocorria um bom trabalho por parte desse profissional. Nas equipes avaliadas, a maioria dos médicos era cubana. Os médicos cubanos vieram para o Brasil em 2013 pelo programa "Mais Médicos", criado pelo governo federal. O projeto tem propostas que objetivariam melhorar a qualidade da política pública de saúde e do SUS. Essa iniciativa levanta temas que merecessem apoio, mas, ao mesmo tempo, traz vários aspectos que não deverão ser apoiados por aqueles interessados no bem-estar dos brasileiros $^{(30)}$. Ainda não se tem estudos que avaliem a atuação desses médicos dentro da ESF, ressaltando, dessa forma, a necessidade de pesquisas que possam verificar o que mudou nesses cenários de práticas.

\section{Caracterização das ações da ESF}

O relato de um enfermeiro demonstra que as atividades são bastante diversificadas e dinâmicas:

\begin{abstract}
"Temos várias atividades na unidade, atuamos como gerente, supervisionamos ACS (Agentes Comunitários de Saúde), realizamos consulta de enfermagem nos programas de saúde da mulher, saúde da criança, hipertensos e diabéticos, tuberculose, hanseniase, realizamos prevenção do CA (câncer) de colo uterino $e$ de mama. Fazemos visitas domiciliares a clientes acamados e puérperas, vacinação, PSE (Programa Saúde na Escola), retiramos pontos, realizamos curativos e atividades educativas. Enfim, muito trabalho burocrático." (ENFERMEIRO 1)
\end{abstract}

Foram estabelecidas algumas diretrizes ou funções que a atenção básica deve cumprir. Dentre elas, está o acolhimento à demanda e busca ativa com avaliação de vulnerabilidade, clínica ampliada e compartilhada, saúde coletiva, participação na gestão e cogestão ${ }^{(31)}$. Percebe-se, pelo relato do enfermeiro, que as ações são muito mais voltadas ao atendimento clínico-ambulatorial, deixando de atender a muitas ações preconizadas. Isso pode ser justificado, dentre outros fatores, pela falta de profissionais e recursos financeiros na rede de atenção básica, realidade também encontrada no estudo realizado em Minas Gerais ${ }^{(10)}$.

\section{Percepção sobre a ESF pelos atores}

Quanto à avaliação da ESF pelos profissionais, a maioria relatou que a organização não é favorável ao trabalho, conforme dito pelos profissionais:

"Tá muito a desejar, não só aqui nessa cidade. Acredito que seja a nivel de Brasil, no sentido de que falta medicação, 
insumos. A gente precisa fazer certas atribuições, e deixa de fazer por conta de materiais que faltam. E a gente tem que tentar trabalhar com o pouco que tem, e o pouco que tem não é satisfatório.” (ENFERMEIRO 4)

"Ainda falta a inserção de algumas equipes para uma nova territorialização e divisão mais igualitária de famílias para os PSF." (ENFERMEIRA 1)

Mesmo com a expansão da ESF em algumas regiões do país, é importante reconhecer que estamos longe de dispor de uma rede de atenção básica com ampla cobertura e eficácia adequada. Verifica-se que há alguns problemas, como o financiamento insuficiente, a ausência de política de pessoal e de um projeto consistente para formação de especialistas $^{(23)}$.

\section{Dificuldades encontradas para a implementação da ESF}

Algumas dificuldades para a implementação da ESF foram citadas pelo gestor. Umas ligadas à infraestrutura, outras ligadas à falta de pessoal para desempenhar algumas funções e outras ligadas à falta de recursos financeiros. Quando foi perguntado à gestora quais eram as principais dificuldades enfrentadas pelo serviço, ela respondeu:

"Falta de recursos humanos e insumos, postos em reforma, não fidelização de carro e motorista para as unidades (...)." (GESTORA)

Gestores de municípios de Minas Gerais afirmaram que as principais limitações que impedem o desenvolvimento da ESF nos municípios são: a incompreensão da população quanto aos objetivos da ESF, a má formação dos profissionais e a dependência dos usuários em relação ao agente comunitário ${ }^{(10)}$.

Serviços de saúde ruins podem causar prejuízos graves para a população. Por isso, esses serviços precisam respeitar normas de qualidade, mas também não devem estar muito formalizados $^{(32)}$.

Geralmente, o setor público de saúde tem poucos recursos financeiros. Isso acaba dando margem para que o gestor público interligue a má qualidade dos serviços prestados à falta de dinheiro para investir nesses serviços. Entretanto, em muitos casos, é possível melhorá-los sem gerar muitos custos. Basta ter um pouco de organização e visão de futuro ${ }^{(33)}$.

A procura pela melhoria do nível de saúde remete à questão da utilização eficiente dos recursos disponíveis para oferecer com eficácia os serviços de saúde. Esse desafio é maior nos países em desenvolvimento, como o Brasil, devido à falta de recursos financeiros, físicos e humanos ${ }^{(20)}$.

Verificou-se, junto aos profissionais, a satisfação e as principais dificuldades no trabalho. A maioria respondeu que se sente valorizada no seu cenário de prática. No entanto, também apontou algumas fragilidades importantes, como o excesso de trabalho por falta de profissionais, baixo salário, falta de infraestrutura e carência de insumos de trabalho, como se observa nas falas a seguir:

\section{"As principais dificuldades encontram-se na carência de insumos e materiais administrativos, dificultando a realização dos programas." (TÉCNICO DE ENFERMAGEM 1)}

“(...) Falta de condições fisicas, estruturais, materiais e medicamentos." (ENFERMEIRO 2)

Percebe-se que as dificuldades estruturais são uma realidade verificada nos estados. Um estudo que avaliou a percepção dos enfermeiros dentro da atenção básica de Fortaleza apontou que essas dificuldades se referem principalmente à própria estrutura física das unidades de saúde, pois algumas não têm medicamentos, material (como bloco de receituário para prescrição) ou transporte. Além disso, a péssima condição das estradas é apontada como empecilho para a realização das visitas, e em muitos lugares, durante a estação chuvosa, somente carros com tração conseguem fazer o percurso, mas estes não estão disponíveis para todas as equipes ${ }^{(34)}$.

É necessário pensar em alguns dilemas para poder refletir sobre o cenário de prática, como: a reordenação do modelo de atenção das UBS tradicionais; a composição mínima que deveria ter as equipes de ESF; a ampliação da multiprofissionalidade com a lógica do apoio matricial; a clientela possível de ser atendida por uma equipe de saúde da família; a relação entre as pessoas, suas famílias e a comunidade; a incorporação do acolhimento sem transformar a ESF em pronto atendimento. Diante disso, seria necessário investigar as dificuldades clínico-gerenciais mais importantes para a qualificação da ESF e, a partir dessa investigação, contribuir para a construção de propostas de formação e cogestão de profissionais capazes de fazer uma clínica mais ampliada ${ }^{(23)}$.

\section{CONSIDERAÇÕES FINAIS}

Há a necessidade de fortalecer a atenção básica localmente, uma vez que a gestora da saúde no município não tinha clareza de como se deu o processo de implementação da ESF. Quanto às atividades desenvolvidas, tem-se no enfermeiro o papel de liderança nas unidades de saúde, sendo responsável tanto pela gestão dos serviços oferecidos quanto pela supervisão dos demais profissionais.

A percepção dos profissionais sobre a Estratégia retrata que bons laços existentes entre os profissionais e a comunidade estavam diretamente ligados à assiduidade 
dos usuários ao serviço. Classificaram como boa a relação entre os membros da equipe de saúde e destacaram a união como seu ponto forte. Relatam também um cenário de incertezas em relação ao futuro, envolvendo a falta de alguns membros na equipe, ou mesmo a falta de equipes completas para suprir a demanda do serviço, bem como limitações quanto à escassez de medicamentos e insumos para a realização de consultas e procedimentos. A falta de recursos humanos e financeiros foi citada, inclusive, como uma dificuldade que impede a plena execução do serviço. E mesmo que algumas profissões se sintam valorizadas, ainda reclamam da carga horária de trabalho exaustiva e da baixa remuneração salarial.

\section{REFERÊNCIAS}

1. Ministério da Saúde (BR). Saúde da família: uma estratégia para a reorganização do modelo assistencial. Brasília: Ministério da Saúde; 1998.

2. Azevedo ALM, Costa AM. A estreita porta de entrada do Sistema Único de Saúde (SUS): uma avaliação do acesso na Estratégia de Saúde da Família. Interface Comun Saúde Educ. 2010;14(35):797-810.

3. Elias PE, Ferreira CW, Alves MCG, Cohn A, Kishima V, Escrivão A Júnior et al. Atenção básica em saúde: comparação entre PSF e UBS por estrato de exclusão social no município de São Paulo. Ciênc Saúde Coletiva. 2006;11(3):633-41.

4. Moimaz SAS, Marques JAM, Saliba O, Garbin CAS, Zina LG, Saliba NA. Satisfação e percepção do usuário do SUS sobre o serviço público de saúde. Physis (Rio J.). 2010;20(4):1419-40.

5. Albuquerque FJB, Melo CF, Souza FE Filho, Araújo JL Neto. Avaliação da estratégia saúde da família a partir das crenças dos profissionais. Estud Psicol (Campinas). 2011;28(3):363-70.

6. Campos FE, Belisário SA. O Programa de Saúde da Família e os desafios para a formação profissional e a educação continuada. Interface Comun Saúde Educ. 2001;5(9):133-41.

7. Ministério da Saúde (BR). Carta dos direitos dos usuários da saúde. Brasília: Ministério da Saúde; 2006.

8. Cecílio LCO, Andreazza R, Souza ALM, Lima MR, Mercadante CEB, Pinto NRS et al. O gestor municipal na atual etapa de implantação do SUS: características e desafios. Reciis. 2007;1(2):1-8.

9. Albanes HC, Lobo E, Bernardini IS. O Programa Nacional de Melhoria do Acesso e da Qualidade da Atenção Básica (PMAQ-AB) e sua relação com a qualidade dos serviços prestados nos centros de saúde. Coleção Gest Saúde Públ. 2013;1(4):83-102.

10. Ronzani TM, Silva CM. O Programa Saúde da Família segundo profissionais de saúde, gestores e usuários. Ciênc Saúde Coletiva. 2008;13(1):23-34.

11. Bardin L. Análise de conteúdo. São Paulo: Edições 70; 2011.

12. Minayo MCS. O desafio do conhecimento: pesquisa qualitativa em saúde. $11^{\mathrm{a}}$ ed. São Paulo: Hucitec Editora; 2014.

13. Trajman A, Assunção N, Venturi M, Tobias D, Toschi W, Brant V. A preceptoria na rede básica da Secretaria Municipal de Saúde do Rio de Janeiro: opinião dos profissionais de Saúde. Rev Bras Educ Med. 2009;33(1):24-32.

14. Machado MH, Oliveira ES, Moyses NMN. Tendências do mercado de trabalho em saúde do Brasil. In: Celia P, Mario RDP, Tania F. O trabalho em saúde: abordagens quantitativas e qualitativas. Rio de Janeiro: CEPESC/ UERJ; 2011. p. 103-16.

15. Lima MCCS, Costa COM, Bigras M, Santana MAO, Alves TDB, Nascimento OC et al. Atuação profissional da atenção básica de saúde face à identificação e notificação da violência infanto-juvenil. Rev Baiana Saúde Pública. 2011;35(1):118-37.

16. Falcão SMR, Sousa MNA. Gerenciamento da atenção primária à saúde: estudo nas unidades básicas. Rev Enferm UFPE. 2011;5(6):1510-7.

17. Santos NR, Amarante PDC. Gestão pública e relação público privado na saúde. Rio de Janeiro: Cebes; 2010.

18. Berretta IQ, Lacerda JT, Calvo MCM. Modelo de avaliação da gestão municipal para o planejamento em saúde. Cad Saúde Pública. 2011;27(11):2143-54.

19. Barreto ML. O conhecimento científico e tecnológico como evidência para políticas e atividades regulatórias em saúde. Ciênc Saúde Coletiva. 2004;9(2):329-38.

20. Erdmann AL, Andrade SR, Mello ALSF, Meirelles BHS. Gestão das práticas de saúde na perspectiva do cuidado complexo. Texto \& Contexto Enferm. 2006;15(3):483-91.

21. Ministério da Saúde (BR). Gestão municipal de saúde: textos básicos. Brasília: Ministério da Saúde, 2001. $344 \mathrm{p}$.

22. Ministério da Saúde (BR). Saúde da família: panorama, avaliação e desafios. $1^{\text {a }}$ ed. Brasília: Ministério da Saúde; 2005. 
23. Campos GWS, Gutiérrez AC, Guerrero A, Cunha GT. Reflexões sobre atenção básica e a Estratégia de Saúde da Família. In: Campos GWS, Guerrero AVP. Manual de práticas de atenção básica: saúde ampliada e compartilhada. $2^{a}$ ed. São Paulo: Hucitec Editora; 2010. p. 132-53.

24. Oliveira EM, Spiri WC. Programa Saúde da Família: a experiência de equipe multiprofissional. Rev Saúde Pública. 2006;40(4):727-33.

25. Alves VS. Um modelo de educação em saúde para o Programa Saúde da Família: pela integralidade da atenção e reorientação do modelo assistencial. Interface Comun Saúde Educ. 2005;9(16):39-52.

26. Ministério da Saúde (BR). Portaria No 648 de 28 de março de 2006. Aprova a Política Nacional de Atenção Básica, estabelecendo a revisão de diretrizes e normas para a organização da atenção básica para o Programa Saúde da Família (PSF) e o Programa Agentes Comunitários de Saúde (PACS). Brasília: Ministério da Saúde; 2006.

27. Nora CRD, Junges JR. Política de humanização na atenção básica: revisão sistemática. Rev Saúde Pública. 2013;47(6):1186-200.

28. Marin MJS, Storniolo LV, Moravcik MY.Ahumanização do cuidado na ótica das equipes da Estratégia de Saúde da Família de um município do interior paulista, Brasil. Rev Latinoam Enferm. 2010;18(4):763-9.
29. Camelo SHH, Pinheiro A, Campos D, Oliveira TL. Auditoria de enfermagem e a qualidade da assistência à saúde: uma revisão da literatura. Rev Eletrônica Enferm. 2009;11(4):1018-25.

30. Campos GWS. A saúde, o SUS e o Programa Mais Médicos. Rev Méd Resid (Online). 2013;15(2):1-4.

31. Campos CEA. O desafio da integralidade segundo as perspectivas da vigilância da saúde e da saúde da família. Ciênc Saúde Coletiva. 2003;8(2):569-84.

32. Shinyashiki GT, Trevisan MA, Mendes IAC. Sobre a criação e a gestão do conhecimento organizacional. Rev Latinoam Enferm. 2003;11(4):499-506.

33. Albarello CB. O papel do administrador na gestão pública. Rev Adm. 2006;5(9):49-71.

34. Medeiros RLR, Andrade AMBA, Fernandes AFC, Almeida NMGS, Lessa MGG. O enfermeiro no Programa Saúde da Família: percepções, possibilidades de atuação, fronteiras profissionais e espaços de negociação. Fortaleza: CETREDE/UFC/UECE, 2007.

\section{Endereço para correspondência:}

Tiago Freire Martins

Rua José Hamilton de Oliveira, 160

Bairro: Centro

CEP: 62930-000 - Limoeiro do Norte - CE - Brasil

E-mail: tiagofreire@yahoo.com.br 